\title{
Pathological Role of Unsaturated Aldehyde Acrolein in Diabetic Retinopathy
}

\author{
Miyuki Murata ${ }^{1,2}, K_{0 u s u k e ~ N o d a}^{1,2 *}$ and Susumu Ishida ${ }^{1,2}$ \\ ${ }^{1}$ Laboratory of Ocular Cell Biology \& Visual Science, Hokkaido University, Sapporo, Japan, ${ }^{2}$ Department of Ophthalmology, \\ Faculty of Medicine and Graduate School of Medicine, Hokkaido University, Sapporo, Japan
}

With increasing prevalence of diabetes and a progressively aging society, diabetic retinopathy is emerging as one of the global leading causes of blindness. Recent studies have shown that vascular endothelial growth factor (VEGF) plays a central role in the pathogenesis of diabetic retinopathy and anti-VEGF agents have become the firstline therapy for the vision-threatening disease. However, recent studies have also demonstrated that diabetic retinopathy is a multifactorial disease and that VEGFindependent mechanism(s) also underlie much of the pathological changes in diabetic retinopathy. Acrolein is a highly reactive unsaturated aldehyde and is implicated in protein

OPEN ACCESS

Edited by:

Sofie Struyf,

KU Leuven, Belgium

Reviewed by:

Mohamed Al-shabrawey, Augusta University, United States

Subhadra Priya Narayanan,

Augusta University, United States

*Correspondence: Kousuke Noda nodako@med.hokudai.ac.jp

Specialty section: This article was submitted to Inflammation,

a section of the journal

Frontiers in Immunology

Received: 30 July 2020 Accepted: 01 October 2020 Published: 22 October 2020

Citation: Murata M, Noda K and Ishida S (2020) Pathological Role of Unsaturated Aldehyde Acrolein in Diabetic Retinopathy.

Front. Immunol. 11:589531. doi: 10.3389/fimmu.2020.589531 dysfunction. As acrolein is common in air pollutants, previous studies have focused on it as an exogenous causative factor, for instance, in the development of respiratory diseases. However, it has been discovered that acrolein is also endogenously produced and induces cell toxicity and oxidative stress in the body. In addition, accumulating evidence suggests that acrolein and/or acrolein-conjugated proteins are associated with the molecular mechanisms in diabetic retinopathy. This review summarizes the pathological roles and mechanisms of endogenous acrolein production in the pathogenesis of diabetic retinopathy.

Keywords: inflammation, oxidative stress, vascular adhesion protein-1, acrolein, diabetic retinopathy, spermine oxidase

\section{INTRODUCTION}

The prevalence of diabetes is increasing worldwide (1) and the global prevalence has been estimated at $10.2 \%$ (578 million) by 2030 and $10.9 \%$ (700 million) by 2045 (2). Diabetic retinopathy (DR) is a retinal microvascular complication caused by diabetes with complex multifactorial pathogenesis. Epidemiological studies have revealed that the prevalence and severity of DR increases with age and duration of diabetes (3). For instance, $23 \%$ of patients with type II diabetes have non-proliferative retinopathy after $11-13$ years, $41 \%$ after $14-16$ years, and $60 \%$ after 16 years (3). Hence, with increasing diabetes prevalence and a progressively aging society, DR is emerging as one of the global leading causes of blindness.

Early events in DR, such as thickening of the capillary basement membrane and pericyte loss, both of which contribute to vascular instability in the diabetic retina, are well known. The changes in retinal microvasculature are followed by capillary non-perfusion and ischemia-mediated pathology 
such as pathologic neovascularization originating from retinal vessels. The pathologic neovascularization due to retinal ischemia causes the formation of fibrovascular tissues at the vitreoretinal surface, which is a hallmark of proliferative diabetic retinopathy (PDR), and leads to severe complications such as vitreous hemorrhage and tractional retinal detachment. Furthermore, retinal ischemia compromises the blood-retinal barrier (BRB) and results in fluid accumulation in the center of the diabetic retina, i.e., diabetic macular edema (DME). During the past few decades extensive efforts have been undertaken to develop therapeutic strategies to control vascular complications in DR.

Recent advances in basic research have demonstrated that vascular endothelial cell growth factor (VEGF) plays a major role in the pathogenesis of DR $(4,5)$ and clinical application of antiVEGF agents have dramatically improved the therapeutic outcomes of the vision-threatening disease. Nowadays, antiVEGF agents have become the standard first-line treatment for $\mathrm{DR}$, in contrast to the past where photocoagulation and vitreous surgery for advanced DR were the only options.

However, accumulating evidence from clinical research has highlighted a patient population with DME that is refractory to anti-VEGF therapy (6). In fact, DME patients who suffer from poor visual acuity caused by sustained exudative changes in the macula despite frequent injections of anti-VEGF agents are often encountered in the clinical setting. This indicates that DR is a multifactorial disease and VEGF-independent mechanisms also underlie many of the pathological changes.

Some of the reported pathological signs in DR include chronic inflammation and oxidative stress (7). Macroscopic signs of inflammation such as redness (Rubor), heat (Calor), swelling (Tumor) and pain (Dolor), are not pathological features in the diabetic retina and the classical definition of inflammation is inadequate to describe the characteristics of DR. However, at a microscopic level, inflammatory responses including vessel dilatation, hemodynamic alteration, exudation and leukocyte accumulation/migration are present in retinal and choroidal tissues during development of DR (8). In addition, there is growing scientific evidence that oxidative stress plays a crucial role in the development of diabetic complications including DR (9).

Therefore, elucidation of "VEGF-independent" pathophysiology in DR is critical to fulfill an unmet medical need for patients with DR refractory to anti-VEGF therapy. In this review, we provide our perspective on the VEGF-independent mechanisms in the pathogenesis of DR, with focus on the unsaturated aldehyde acrolein.

\section{WHAT IS ACROLEIN?}

Acrolein is a highly reactive unsaturated aldehyde that causes protein dysfunction by reacting preferentially with Cys, Lys and His residues of peptide chains via Michael-type reaction $(10,11)$ (Figure 1A). In acrolein-conjugated proteins, the Lys adduct is a more stable product than the other adducts. One of the major acrolein-Lys adducts, $\mathrm{N}^{\mathrm{e}}$-(3-formyl-3, 4-dehydropiperidino) lysine adduct (FDP-Lys) (Figure 1B), is known as a reactive intermediate that can covalently bind to thiols, including glutathione (GSH), through the retained electrophilic carbonyl moiety (10). Since the reaction of GSH with acrolein and/or FDP-Lys depletes intracellular reserves of GSH, which is a major antioxidant enzyme, acrolein increases oxidative stress by limiting oxidative stress resistance in the body (12). Notably, previous studies have implicated acrolein in a wide range of vascular diseases such as brain infarction (13) and neurodegenerative diseases such as Parkinson's disease (14) and Alzheimer's disease (15). Since DR is a representative retinal disease with characteristics of both vascular disease and neurodegenerative disease, in recent years much attention has been paid to acrolein as a possible participant in the pathogenesis of DR.

\section{ENDOGENOUS ACROLEIN GENERATION}

Since acrolein is common in air pollutants such as cigarette smoke, vehicle exhaust and overheated cooking oil, acrolein was previously focused on as an exogenous causative substance in the research of respiratory diseases such as lung cancer (16). However, recent studies including ours have revealed that acrolein is also generated endogenously through peroxidation of unsaturated fatty acids (17) and polyamine metabolism (18), resulting in cell toxicity and oxidative stress. Among the molecules related to endogenous acrolein generation, the current review focuses on two major enzymes, both of which are implicated in the development of DR.

\section{Vascular Adhesion Protein-1 (VAP-1)}

Vascular adhesion protein-1 (VAP-1) is a homodimeric sialylated glycoprotein expressed in vascular endothelial cells and involved in leukocyte transmigration (19-21). Previous studies have elucidated that VAP-1 is crucial in the pathology of systemic inflammatory diseases, including rheumatoid arthritis $(22,23)$, inflammatory bowel diseases $(24)$, myocardial<smiles>C=CC=O</smiles>

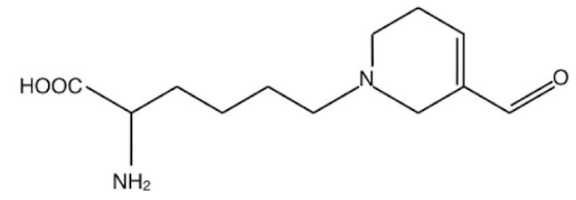

FIGURE 1 | Structures of (A) acrolein and (B) one of the major acrolein-conjugated proteins, $\mathrm{N}^{e}$-(3-formyl-3, 4-dehydropiperidino) lysine adduct (FDP-Lys). 
infarction (25), and diabetes (26). In ocular tissues, we reported that VAP-1 is localized to endothelial cells of retinal and choroidal vessels (27), and VAP-1 is involved in the molecular mechanisms of acute ocular inflammation and inflammationassociated ocular angiogenesis $(28,29)$. In addition, we also showed that VAP-1 blockade significantly reduced the transmigration and capillary entrapment of leukocytes in the retina in a diabetic animal model (30). Taken together, our prior research demonstrates that VAP-1 plays a role in the pathogenesis of DR by mediating leukocyte recruitment as a leukocyte adhesion molecule.

VAP-1 also exists as a soluble form (sVAP-1) in mammals and is known to participate in theinitiation and development of systemic disorders (31-33). In addition to its role as anadhesion molecule, both membrane and soluble forms of VAP-1 function as semicarbazide-sensitiveamine oxidase (SSAO), which oxidizes aliphatic and aromatic primary monoamines and converts them tothe corresponding aldehydes with the release of hydrogen peroxide and ammonia (Figure 2) (34). Of note, oursubsequent analyses revealed that high glucose, inflammatory cytokines such as tumor necrosisfactor- $\alpha$ (TNF- $\alpha$ ) and interleukin-1 $\beta$ (IL-1 $\beta$ ) and angiogenic factor VEGF facilitate proteolytic cleavage of the membrane-bound VAP-1 from retinal capillary endothelial cells mediated by matrix metalloproteinase (MMP)-2 and MMP-9 (35, 36), both of which are important in fibrovascular tissue formation (Figure 3) (37). In addition, our in vitro study revealed that SVAP1 mediates acrolein production via spermine metabolism, a polyamine in retinal endothelial cells (18). Polyamines are low molecular weight polycations that have two or more primary amine groups, and are known to play an important role in cell proliferation and differentiation (38). In mammals, there are three naturally occurring polyamines: putrescine, spermidine, and spermine (39). In patients with PDR, spermine levels are elevated 15-fold in the vitreous fluid compared to non-DR patients (40), indicating that the vitreous cavity is a substrateenriched environment for sVAP-1. Indeed, sVAP-1 and FDP-Lys are increased and are correlated in the vitreous fluid of patients with PDR (18). Evidence suggests that acrolein is generated by intravitreal sVAP-1 released from retinal capillary endothelial cells in the presence of participant molecules such as inflammatory cytokines and proteinases in eyes with DR.

\section{Spermine Oxidase}

Spermine oxidase (SMOX) is a flavin adenine dinucleotidecontaining enzyme that catalyzes the oxidative degradation of spermine to produce spermidine, hydrogen peroxides, and 3 -aminopropanal (41) that is non-enzymatically converted to acrolein (Figure 4) (42). SMOX is a highly inducible enzyme and its expression is upregulated by inflammatory cytokines, including TNF- $\alpha$ and IL-6 $(43,44)$, both of which are increased in eyes with PDR. Recent studies have revealed that FDP-Lys is accumulated in Müller glial cells of streptozotocin (STZ)-induced diabetic rats (45) and in migrated glial cells in the fibrovascular tissues obtained from patients with PDR (46). Therefore, both observation in humans and experimental evidence indicate that acrolein is generated and/or accumulates in retinal glial cells under diabetic conditions. However, retinal glial cells lack VAP-1 expression, and the exact mechanism of acrolein generation in these cells was unclear. Recently, our group discovered that SMOX mediates acrolein generation in cultured retinal glial cells, and that hypoxia induces SMOX production via HIF-1 binding to SMOX promoter (47). In addition, the localization of SMOX was seen predominantly in glial cells of fibrovascular tissues (47). In the advanced stage of $\mathrm{DR}$, obliteration of retinal microvasculature elicits a decrease in tissue oxygen concentration $(48,49)$ and tissue hypoxia induces extensive cellular responses, including neovascularization that eventually results in proliferative changes at the vitreoretinal surface in eyes with DR. Our in vitro study revealed that hypoxia increases FDP-Lys and hydrogen peroxide levels in cultured retinal glial cells, which were abrogated by the potent SMOX inhibitor MDL72527 (47). Overall, the experimental evidence indicates that SMOX produces acrolein through the enzymatic conversion of spermine to spermidine in retinal glial cells under hypoxic conditions and presumably exacerbates oxidative stress in eyes with DR.
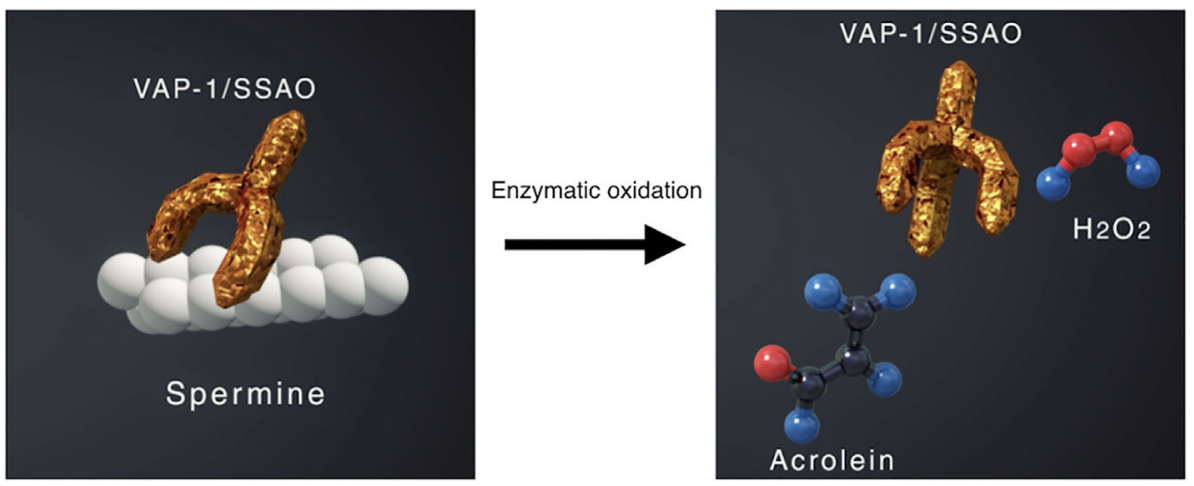

FIGURE 2 | Enzymatic oxidation of spermine mediated by vascular adhesion protein-1 (VAP-1) function as semicarbazide-sensitive amine oxidase (SSAO). VAP-1 converts spermine to acrolein and hydrogen peroxide, both of which increase oxidative stress in the body. 

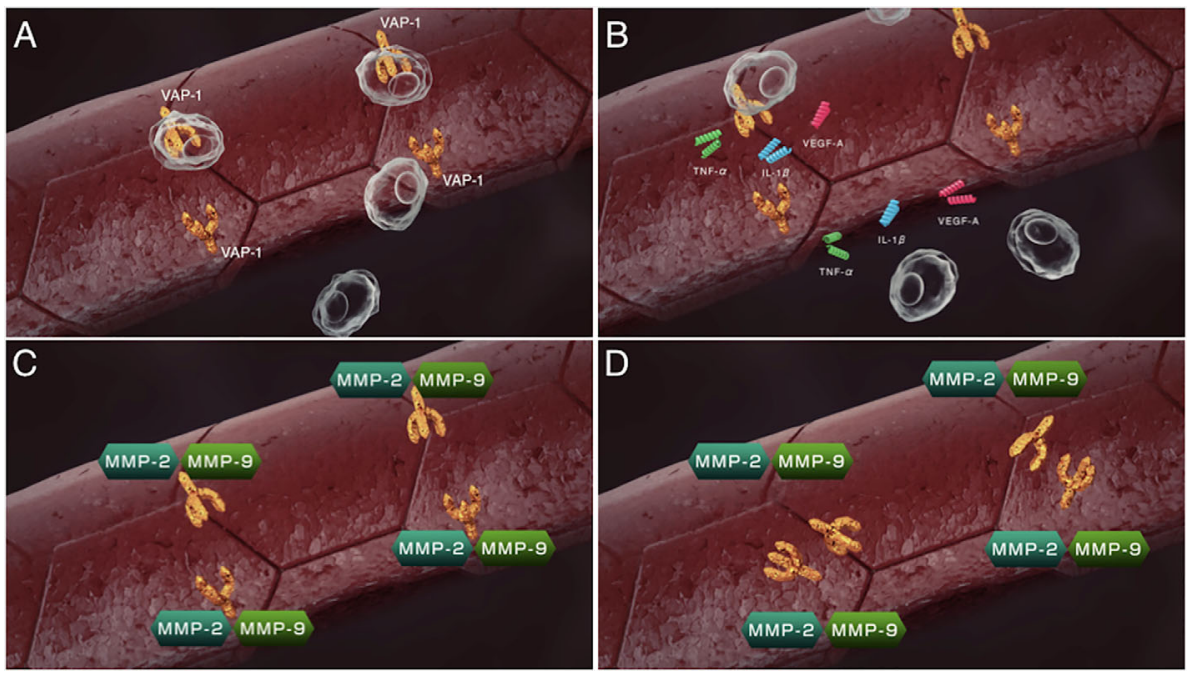

FIGURE 3 | Sequential steps of release of the soluble form of VAP-1 from retinal capillary endothelial cells. (A) VAP-1 and other leukocyte adhesion molecules facilitate leukocyte recruitment upon inflammation. (B) Recruited leukocytes secrete inflammatory cytokines such as tumor necrosis factor- $\alpha$ (TNF- $\alpha$ ), interleukin$1 \beta(I L-1 \beta)$ and vascular endothelial cell growth factor (VEGF). (C) MMP-2 and MMP-9 induced by the inflammatory cytokines proteolytically cleave the VAP-1 protein. (D) Soluble form of VAP-1 was released from the surface of endothelial cells.

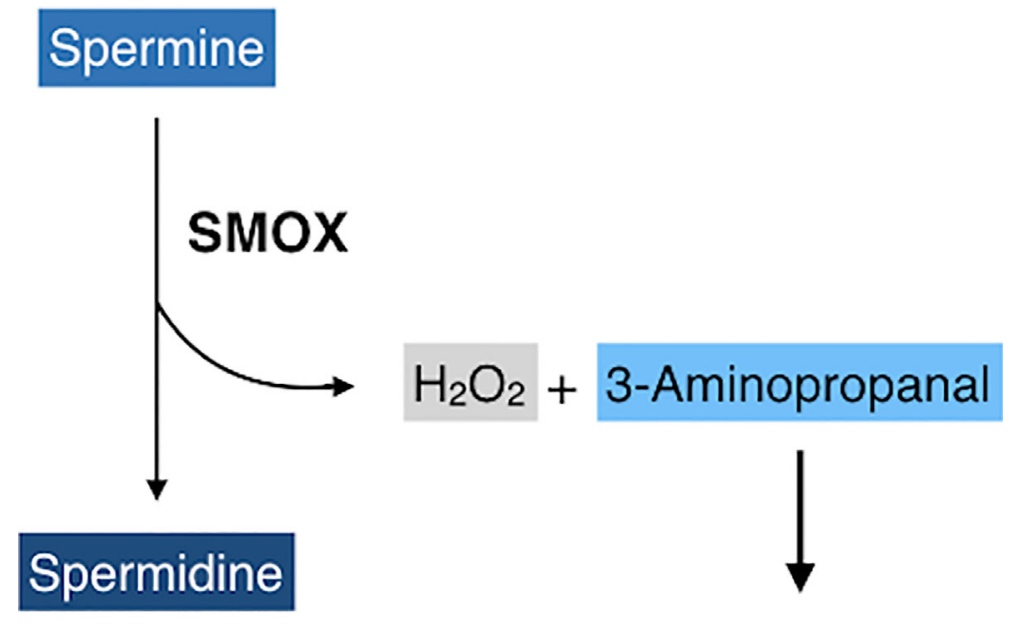

Acrolein

FIGURE 4 | A schematic of oxidative degradation of spermine by spermine oxidase (SMOX). SMOX catalyzes spermine and produces spermidine, hydrogen peroxides, and 3-aminopropanal, which is non-enzymatically converted to acrolein.

\section{ROLES OF ACROLEIN IN DR}

So far, it has been demonstrated that acrolein plays a significant role in the pathogenesis of systemic disorders, such as neurodegenerative diseases (50), cardiovascular diseases (51), and diabetes (52). With respect to diabetes, acrolein was reportedly increased in the serum and urine of patients with diabetes $(52,53)$. In the eye, previous studies demonstrated that
FDP-Lys markedly increased in retinal glial cells of experimental diabetic rodents $(45,54,55)$. We also reported that FDP-Lys was elevated in the vitreous fluid of patients with PDR (18). In addition, we found that FDP-Lys largely accumulated in glial cells (46) and endothelial cells (18) of fibrovascular tissues obtained from patients with PDR. Therefore, these data suggest that acrolein participates in the development of DR. 


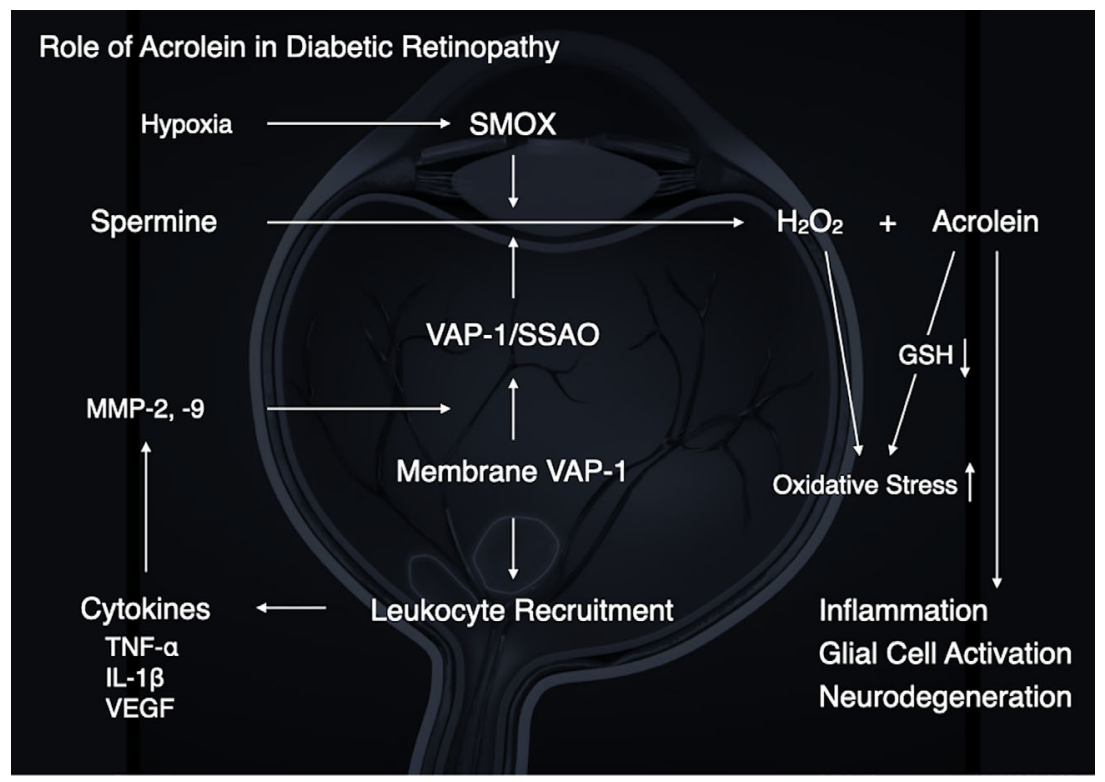

FIGURE 5 | A schematic summary illustrating the role of acrolein in diabetic retinopathy.

In the following section, we summarize the role of acrolein in the pathogenesis of DR.

\section{Inflammation}

Recent studies have shown that chronic, low-grade inflammation underlies much of the vascularcomplications of DR $(56,57)$. Previously, it was demonstrated that acrolein activated the NF$\kappa$ Bpathway and induced pro-inflammatory cytokines including cyclooxygenase-2 (58). In addition, it was shown that acrolein induced pulmonary inflammation anddeath of lung epithelial cells via induction of NF- $\mathrm{KB}$ signaling in a mouse in vivo study (59). Acrolein also induced TNF- $\alpha, I L-6$, and $I L-8$ mRNA expression through NF- $\kappa \mathrm{B}$ activation in human umbilical vein endothelial cells (HUVECs) (60).

In the retina, it was reported that acrolein induced transforming growth factor beta-1(TGF $\beta 1$ ), TGF 32 , and VEGF production in retinal pigment epithelium inhyperglycemic conditions (61). In contrast, anacrolein-scavenging agent significantly suppressed C-C motif chemokine ligand $2(\mathrm{Ccl} 2)$, $I L-1 \beta$, and intercellular adhesion molecule 1 (Icam1) mRNA expression in the retina of diabetic rats (55). Accumulating evidence clearly indicates the involvement of acrolein in the inflammatory aspect of DR; however, further analysis is needed for the association between acrolein and pro-inflammatory cytokine production in DR.

\section{Increase in Oxidative Stress}

The role of oxidative stress in the pathogenesis of DR has been extensively investigated in experimental and clinical studies. The levels of various reactive oxygen species (ROS), including superoxide (62) and hydrogen peroxide (63), have been shown to be elevated in the retina of diabetic animals. Conversely, the antioxidant defense systems in eyes of patients with DR have been shown to be functionally damaged (64). We previously demonstrated that acrolein reduces the intracellular reserves of GSH, leading to consequent increase in oxidative stress and cell death in retinal microvascular endothelial cells and glial cells $(18,65)$. Furthermore, the subsequent analysis further revealed that spermine oxidation by VAP-1 or SMOX results in acrolein generation and exacerbates oxidative stress in the microenvironment by both limiting the anti-oxidant defense system and enhancing ROS generation $(18,47)$. As aforementioned, VAP-1 and FDP-Lys are increased and are correlated in the vitreous fluid of patients with PDR (18). VAP-1 and $\mathrm{N}$ epsilon-(hexanoyl)lysine (HEL), an oxidative stress marker, are also correlated in the vitreous fluid from PDR patients (35). These data indicate that increased acrolein generated by VAP-1 and SMOX participates in the pathogenesis of DR.

\section{Glial Cell Activation}

Glial cell activation is the initial response during the early stages of DR $(66,67)$ and retinal glial cells are known to proliferate and migrate into the vitreoretinal interface, which eventually leads to fibrovascular tissue formation during PDR progression $(68,69)$. Acrolein stimulated cell migration of Müller glial cells through induction of C-X-C motif chemokine ligand 1 (CXCL1) protein (65), a member of the C-X-C family of chemokines that promotes neutrophil and tumor cell migration through binding to the receptor C-X-C motif chemokine receptor 2 (CXCR2) (69, 70). It has been previously shown that the vitreous level of CXCL1 was increased in eyes affected by PDR (71) and in the retinal tissue of diabetic mice (72). In addition, we reported that CXCL1 was localized in GFAP-positive cells of fibrovascular tissues (65). Since FDP-Lys largely accumulates in the glial cells of fibrovascular tissues (46), it suggests that CXCL1 stimulates 
retinal glial cells in an autocrine fashion through its receptor, CXCR2, in response to acrolein under diabetic conditions (65). Previously, it was reported that an acrolein-scavenging agent could suppress glial fibrillary acidic protein (GFAP) expression, a representative marker of glial cell activation, in Müller glial cells of diabetic rats (55). Therefore, these data indicate that acrolein is potentially one of the molecules triggering retinal glial cell activation in diabetic eyes.

\section{Neurodegeneration}

The retinal tissue consists of vascular and neural components. Whereas microvascular changes are integral to the formation and devastating effects of DR, DR has been recently recognized as a neuro-vascular disease. There is a growing body of evidence to indicate that neural components are damaged in the early phase of DR, even in the absence of vascular complications such as retinal hemorrhage and microaneurysm formation $(73,74)$. Basic research findings obtained from postmortem human specimens (75) and diabetic animals showed that diabetes causes cellular disturbance in neural cells, particularly in retinal ganglion cells (RGC) (76).

As mentioned, SMOX produces acrolein through its enzymatic activity. Liu et al. reported elevated levels of FDPLys in the ganglion cell layer and inner nuclear layer of the retina in a diabetic animal model (77). It was shown that SMOX inhibitor MDL72527 reduced the upregulation of FDP-Lys, retinal tissue thinning and RGC loss (77), suggesting that acrolein is potentially involved in the neurodegeneration in diabetic eyes.

\section{THERAPEUTIC APPROACHES TARGETING ACROLEIN}

As described thus far, acrolein plays a significant role in DR and may be an important target for the prevention and treatment of DR. 2-Mercaptoethanesulfonate (MESNA) is a potent thiol based scavenger of acrolein that has already been used clinically to prevent urothelial toxicity by inactivating metabolites from antineoplastic agents, such as ifosfamide or cyclophosphamide (78). It was also reported that acrolein-scavenging agent 2-hydrazino-4,6-

\section{REFERENCES}

1. Amos AF, McCarty DJ, Zimmet P. The rising global burden of diabetes and its complications: estimates and projections to the year 2010. Diabetes Med (1997) 14 Suppl 5:S1-85. doi: 10.1002/(SICI)1096-9136(199712)14:5+<S7:: AID-DIA522>3.3.CO;2-I

2. Saeedi P, Petersohn I, Salpea P, Malanda B, Karuranga S, Unwin N, et al. Global and regional diabetes prevalence estimates for 2019 and projections for 2030 and 2045: Results from the International Diabetes Federation Diabetes Atlas, 9(th) edition. Diabetes Res Clin Pract (2019) 157:107843. doi: 10.1016/ j.diabres.2019.107843

3. Negi A, Vernon SA. An overview of the eye in diabetes. J R Soc Med (2003) 96 (6):266-72. doi: 10.1258/jrsm.96.6.266

4. Adamis AP, Miller JW, Bernal MT, D’Amico DJ, Folkman J, Yeo TK, et al. Increased vascular endothelial growth factor levels in the vitreous of eyes with dimethylpyrimidine (2-HDP) reduced Müller cell gliosis and retinal inflammatory marker expression in STZ-induced diabetic rats (55). Alternatively, SMOX inhibitor MDL72527 treatment improved electroretinogram response and reduced retinal neurodegeneration in STZ-induced diabetic mice $(77,79)$. Therefore, inhibitors of acrolein and SMOX are potential therapeutic drugs for the treatment of DR.

\section{CONCLUSIONS}

Previous studies have shown that levels of inflammatory cytokines and oxidative stress markers are elevated in the specimens of patients with DR, indicating the roles of the inflammatory process and oxidative stress in retinal microvascular complications caused by diabetes.

The pathogenesis of DR is not entirely known. However, based on the preceding discussion, growing evidence suggests a pathological role for acrolein in the development of DR (Figure 5). Our group and others have demonstrated that diabetic conditions, which include high glucose, inflammation and hypoxia, enhance acrolein production through VAP-1/SSAO and SMOX induction, suggesting that acrolein contributes to the exacerbation of DR via enhancement of inflammation, oxidative stress, glial activation, and neurodegeneration.

\section{AUTHOR CONTRIBUTIONS}

MM wrote the paper. KN wrote and revised the paper. SI revised the paper. All authors contributed to the article and approved the submitted version.

\section{FUNDING}

This work was supported by Grant-in-Aid for Scientific Research (B) $[20 \mathrm{H} 03837(\mathrm{SI})]$ and Grant-in-Aid for Scientific Research (C) [18K09393(KN) and 17K11442(MM)] of the Japan Society for the Promotion of Science. 
retinopathy in Type 2 diabetes. Diabetes Med (2007) 24(9):969-76. doi: 10.1111/ j.1464-5491.2007.02217.x

9. Hammes HP. Diabetic retinopathy: hyperglycaemia, oxidative stress and beyond. Diabetologia (2018) 61(1):29-38. doi: 10.1007/s00125-017-4435-8

10. Aldini G, Orioli M, Carini M. Protein modification by acrolein: relevance to pathological conditions and inhibition by aldehyde sequestering agents. Mol Nutr Food Res (2011) 55(9):1301-19. doi: 10.1002/mnfr.201100182

11. Stevens JF, Maier CS. Acrolein: sources, metabolism, and biomolecular interactions relevant to human health and disease. Mol Nutr Food Res (2008) 52(1):7-25. doi: 10.1002/mnfr.200700412

12. Luo J, Shi R. Acrolein induces oxidative stress in brain mitochondria. Neurochem Int (2005) 46(3):243-52. doi: 10.1016/j.neuint.2004.09.001

13. Saiki R, Park H, Ishii I, Yoshida M, Nishimura K, Toida T, et al. Brain infarction correlates more closely with acrolein than with reactive oxygen species. Biochem Biophys Res Commun (2011) 404(4):1044-9. doi: 10.1016/ j.bbrc.2010.12.107

14. Ambaw A, Zheng L, Tambe MA, Strathearn KE, Acosta G, Hubers SA, et al. Acrolein-mediated neuronal cell death and alpha-synuclein aggregation: Implications for Parkinson's disease. Mol Cell Neurosci (2018) 88:70-82. doi: 10.1016/j.mcn.2018.01.006

15. Dang TN, Arseneault M, Murthy V, Ramassamy C. Potential role of acrolein in neurodegeneration and in Alzheimer's disease. Curr Mol Pharmacol (2010) 3(2):66-78. doi: 10.2174/1874-470211003020066

16. Feng $\mathrm{Z}, \mathrm{Hu} \mathrm{W}, \mathrm{Hu} \mathrm{Y}$, Tang MS. Acrolein is a major cigarette-related lung cancer agent: Preferential binding at p53 mutational hotspots and inhibition of DNA repair. Proc Natl Acad Sci U.S.A. (2006) 103(42):15404-9. doi: 10.1073/pnas.0607031103

17. Uchida K, Kanematsu M, Morimitsu Y, Osawa T, Noguchi N, Niki E. Acrolein is a product of lipid peroxidation reaction. Formation of free acrolein and its conjugate with lysine residues in oxidized low density lipoproteins. J Biol Chem (1998) 273(26):16058-66. doi: 10.1074/jbc.273.26.16058

18. Murata M, Noda K, Kawasaki A, Yoshida S, Dong Y, Saito M, et al. Soluble Vascular Adhesion Protein-1 Mediates Spermine Oxidation as SemicarbazideSensitive Amine Oxidase: Possible Role in Proliferative Diabetic Retinopathy. Curr Eye Res (2017) 42(12):1674-83. doi: 10.1080/02713683.2017.1359847

19. Salmi M, Jalkanen S. A 90-kilodalton endothelial cell molecule mediating lymphocyte binding in humans. Science (1992) 257(5075):1407-9. doi: 10.1126/science. 1529341

20. Salmi M, Jalkanen S. VAP-1: an adhesin and an enzyme. Trends Immunol (2001) 22(4):211-6. doi: 10.1016/s1471-4906(01)01870-1

21. Noda K, Nakao S, Ishida S, Ishibashi T. Leukocyte adhesion molecules in diabetic retinopathy. J Ophthalmol (2012) 2012:279037. doi: 10.1155/2012/ 279037

22. Salmi M, Kalimo K, Jalkanen S. Induction and function of vascular adhesion protein-1 at sites of inflammation. J Exp Med (1993) 178(6):2255-60. doi: 10.1084/jem.178.6.2255

23. Akin E, Aversa J, Steere AC. Expression of adhesion molecules in synovia of patients with treatment-resistant lyme arthritis. Infect Immun (2001) 69 (3):1774-80. doi: 10.1128/IAI.69.3.1774-1780.2001

24. Liaskou E, Karikoski M, Reynolds GM, Lalor PF, Weston CJ, Pullen N, et al. Regulation of mucosal addressin cell adhesion molecule 1 expression in human and mice by vascular adhesion protein 1 amine oxidase activity. Hepatology (2011) 53(2):661-72. doi: 10.1002/hep.24085

25. Jaakkola K, Jalkanen S, Kaunismaki K, Vanttinen E, Saukko P, Alanen K, et al. Vascular adhesion protein-1, intercellular adhesion molecule-1 and P-selectin mediate leukocyte binding to ischemic heart in humans. J Am Coll Cardiol (2000) 36(1):122-9. doi: 10.1016/s0735-1097(00)00706-3

26. Boomsma F, van den Meiracker AH, Winkel S, Aanstoot HJ, Batstra MR, Man in 't Veld AJ, et al. Circulating semicarbazide-sensitive amine oxidase is raised both in type I (insulin-dependent), in type II (non-insulin-dependent) diabetes mellitus and even in childhood type I diabetes at first clinical diagnosis. Diabetologia (1999) 42(2):233-7. doi: 10.1007/s001250051143

27. Almulki L, Noda K, Nakao S, Hisatomi T, Thomas KL, Hafezi-Moghadam A. Localization of vascular adhesion protein-1 (VAP-1) in the human eye. Exp Eye Res (2010) 90(1):26-32. doi: 10.1016/j.exer.2009.09.005

28. Noda K, Miyahara S, Nakazawa T, Almulki L, Nakao S, Hisatomi T, et al. Inhibition of vascular adhesion protein-1 suppresses endotoxin-induced uveitis. FASEB J (2008) 22(4):1094-103. doi: 10.1096/fj.07-9377com
29. Noda K, She H, Nakazawa T, Hisatomi T, Nakao S, Almulki L, et al. Vascular adhesion protein-1 blockade suppresses choroidal neovascularization. FASEB $J$ (2008) 22(8):2928-35. doi: 10.1096/fj.07-105346

30. Noda K, Nakao S, Zandi S, Engelstadter V, Mashima Y, Hafezi-Moghadam A. Vascular adhesion protein-1 regulates leukocyte transmigration rate in the retina during diabetes. Exp Eye Res (2009) 89(5):774-81. doi: 10.1016/ j.exer.2009.07.010

31. Aalto K, Maksimow M, Juonala M, Viikari J, Jula A, Kahonen M, et al. Soluble vascular adhesion protein-1 correlates with cardiovascular risk factors and early atherosclerotic manifestations. Arterioscler Thromb Vasc Biol (2012) 32 (2):523-32. doi: 10.1161/ATVBAHA.111.238030

32. Hernandez-Guillamon M, Sole M, Delgado P, Garcia-Bonilla L, Giralt D, Boada C, et al. VAP-1/SSAO plasma activity and brain expression in human hemorrhagic stroke. Cerebrovasc Dis (2012) 33(1):55-63. doi: 10.1159/ 000333370

33. Weston CJ, Shepherd EL, Claridge LC, Rantakari P, Curbishley SM, Tomlinson JW, et al. Vascular adhesion protein-1 promotes liver inflammation and drives hepatic fibrosis. J Clin Invest (2015) 125(2):50120. doi: $10.1172 /$ JCI73722

34. Salmi M, Jalkanen S. Cell-surface enzymes in control of leukocyte trafficking. Nat Rev Immunol (2005) 5(10):760-71. doi: 10.1038/nri1705

35. Murata M, Noda K, Fukuhara J, Kanda A, Kase S, Saito W, et al. Soluble vascular adhesion protein-1 accumulates in proliferative diabetic retinopathy. Invest Ophthalmol Vis Sci (2012) 53(7):4055-62. doi: 10.1167/iovs.12-9857

36. Yoshida S, Murata M, Noda K, Matsuda T, Saito M, Saito W, et al. Proteolytic cleavage of vascular adhesion protein-1 induced by vascular endothelial growth factor in retinal capillary endothelial cells. Jpn J Ophthalmol (2018) 62(2):256-64. doi: 10.1007/s10384-017-0555-4

37. Noda K, Ishida S, Inoue M, Obata K, Oguchi Y, Okada Y, et al. Production and activation of matrix metalloproteinase- 2 in proliferative diabetic retinopathy. Invest Ophthalmol Vis Sci (2003) 44(5):2163-70. doi: 10.1167/iovs.02-0662

38. Heby O. Role of polyamines in the control of cell proliferation and differentiation. Differentiation (1981) 19(1):1-20. doi: 10.1111/j.14320436.1981.tb01123.x

39. Pegg AE. Toxicity of polyamines and their metabolic products. Chem Res Toxicol (2013) 26(12):1782-800. doi: 10.1021/tx400316s

40. Nicoletti R, Venza I, Ceci G, Visalli M, Teti D, Reibaldi A. Vitreous polyamines spermidine, putrescine, and spermine in human proliferative disorders of the retina. Br J Ophthalmol (2003) 87(8):1038-42. doi: 10.1136/ bjo.87.8.1038

41. Sharmin S, Sakata K, Kashiwagi K, Ueda S, Iwasaki S, Shirahata A, et al. Polyamine cytotoxicity in the presence of bovine serum amine oxidase. Biochem Biophys Res Commun (2001) 282(1):228-35. doi: 10.1006/ bbrc.2001.4569

42. Cervelli M, Leonetti A, Cervoni L, Ohkubo S, Xhani M, Stano P, et al. Stability of spermine oxidase to thermal and chemical denaturation: comparison with bovine serum amine oxidase. Amino Acids (2016) 48(10):2283-91. doi: 10.1007/s00726-016-2273-5

43. Babbar N, Casero RAJr. Tumor necrosis factor-alpha increases reactive oxygen species by inducing spermine oxidase in human lung epithelial cells: a potential mechanism for inflammation-induced carcinogenesis. Cancer Res (2006) 66(23):11125-30. doi: 10.1158/0008-5472.CAN-06-3174

44. Cervelli M, Bellavia G, Fratini E, Amendola R, Polticelli F, Barba M, et al. Spermine oxidase (SMO) activity in breast tumor tissues and biochemical analysis of the anticancer spermine analogues BENSpm and CPENSpm. BMC Cancer (2010) 10:555. doi: 10.1186/1471-2407-10-555

45. Yong PH, Zong H, Medina RJ, Limb GA, Uchida K, Stitt AW, et al. Evidence supporting a role for $\mathrm{N}$-(3-formyl-3,4-dehydropiperidino)lysine accumulation in Muller glia dysfunction and death in diabetic retinopathy. Mol Vision (2010) 16:2524-38.

46. Dong Y, Noda K, Murata M, Yoshida S, Saito W, Kanda A, et al. Localization of Acrolein-Lysine Adduct in Fibrovascular Tissues of Proliferative Diabetic Retinopathy. Curr Eye Res (2017) 42(1):111-7. doi: 10.3109/ 02713683.2016.1150491

47. Wu D, Noda K, Murata M, Liu Y, Kanda A, Ishida S. Regulation of Spermine Oxidase through Hypoxia-Inducible Factor-1alpha Signaling in Retinal Glial Cells under Hypoxic Conditions. Invest Ophthalmol Vis Sci (2020) 61(6):52. doi: $10.1167 /$ iovs.61.6.52 
48. Frank RN. Diabetic retinopathy. N Engl J Med (2004) 350(1):48-58. doi: 10.1056/NEJMra021678

49. Sutherland FS, Stefansson E, Hatchell DL, Reiser H. Retinal oxygen consumption in vitro. The effect of diabetes mellitus, oxygen and glucose. Acta Ophthalmol (Copenh) (1990) 68(6):715-20. doi: 10.1111/j.17553768.1990.tb01701.x

50. Wood PL, Khan MA, Moskal JR. The concept of "aldehyde load" in neurodegenerative mechanisms: cytotoxicity of the polyamine degradation products hydrogen peroxide, acrolein, 3-aminopropanal, 3 acetamidopropanal and 4-aminobutanal in a retinal ganglion cell line. Brain Res (2007) 1145:150-6. doi: 10.1016/j.brainres.2006.10.004

51. DeJarnett N, Conklin DJ, Riggs DW, Myers JA, O’Toole TE, Hamzeh I, et al. Acrolein exposure is associated with increased cardiovascular disease risk. J Am Heart Assoc (2014) 3(4):e000934. doi: 10.1161/JAHA.114.000934

52. Daimon M, Sugiyama K, Kameda W, Saitoh T, Oizumi T, Hirata A, et al. Increased urinary levels of pentosidine, pyrraline and acrolein adduct in type 2 diabetes. Endocr J (2003) 50(1):61-7. doi: 10.1507/endocri.50.61

53. Ali MF, Kishikawa N, Ohyama K, Mohamed HA, Abdel-Wadood HM, Mahmoud AM, et al. Chromatographic determination of low-molecular mass unsaturated aliphatic aldehydes with peroxyoxalate chemiluminescence detection after fluorescence labeling with 4-(N,N-dimethylaminosulfonyl)-7hydrazino-2,1,3-benzoxadiazole. J Chromatogr B Analyt Technol BioMed Life Sci (2014) 953-954:147-52. doi: 10.1016/j.jchromb.2014.02.009

54. Curtis TM, Hamilton R, Yong PH, McVicar CM, Berner A, Pringle R, et al. Muller glial dysfunction during diabetic retinopathy in rats is linked to accumulation of advanced glycation end-products and advanced lipoxidation end-products. Diabetologia (2011) 54(3):690-8. doi: 10.1007/ s00125-010-1971-x

55. McDowell RE, Barabas P, Augustine J, Chevallier O, McCarron P, Chen M, et al. Muller glial dysfunction during diabetic retinopathy in rats is reduced by the acrolein-scavenging drug, 2-hydrazino-4,6-dimethylpyrimidine. Diabetologia (2018) 61(12):2654-67. doi: 10.1007/s00125-018-4707-y

56. Antonetti DA, Barber AJ, Bronson SK, Freeman WM, Gardner TW, Jefferson LS, et al. Diabetic retinopathy: seeing beyond glucose-induced microvascular disease. Diabetes (2006) 55(9):2401-11. doi: 10.2337/db05-1635

57. Gardner TW, Antonetti DA, Barber AJ, LaNoue KF, Levison SW. Diabetic retinopathy: more than meets the eye. Surv Ophthalmol (2002) 47 Suppl 2: S253-62. doi: 10.1016/s0039-6257(02)00387-9

58. Sarkar $\mathrm{P}$, Hayes BE. Induction of COX-2 by acrolein in rat lung epithelial cells. Mol Cell Biochem (2007) 301(1-2):191-9. doi: 10.1007/s11010-007-9411-z

59. Sun Y, Ito S, Nishio N, Tanaka Y, Chen N, Isobe K. Acrolein induced both pulmonary inflammation and the death of lung epithelial cells. Toxicol Lett (2014) 229(2):384-92. doi: 10.1016/j.toxlet.2014.06.021

60. Haberzettl P, Vladykovskaya E, Srivastava S, Bhatnagar A. Role of endoplasmic reticulum stress in acrolein-induced endothelial activation. Toxicol Appl Pharmacol (2009) 234(1):14-24. doi: 10.1016/j.taap.2008.09.019

61. Grigsby J, Betts B, Vidro-Kotchan E, Culbert R, Tsin A. A possible role of acrolein in diabetic retinopathy: involvement of a VEGF/TGFbeta signaling pathway of the retinal pigment epithelium in hyperglycemia. Curr Eye Res (2012) 37(11):1045-53. doi: 10.3109/02713683.2012.713152

62. Du Y, Miller CM, Kern TS. Hyperglycemia increases mitochondrial superoxide in retina and retinal cells. Free Radic Biol Med (2003) 35 (11):1491-9. doi: 10.1016/j.freeradbiomed.2003.08.018

63. Ellis EA, Guberski DL, Somogyi-Mann M, Grant MB. Increased H2O2, vascular endothelial growth factor and receptors in the retina of the BBZ/ Wor diabetic rat. Free Radic Biol Med (2000) 28(1):91-101. doi: 10.1016/ s0891-5849(99)00216-6

64. Kowluru RA, Chan PS. Oxidative stress and diabetic retinopathy. Exp Diabetes Res (2007) 2007:43603. doi: 10.1155/2007/43603
65. Murata M, Noda K, Yoshida S, Saito M, Fujiya A, Kanda A, et al. Unsaturated Aldehyde Acrolein Promotes Retinal Glial Cell Migration. Invest Ophthalmol Vis Sci (2019) 60(13):4425-35. doi: 10.1167/iovs.19-27346

66. Vujosevic S, Micera A, Bini S, Berton M, Esposito G, Midena E. Aqueous Humor Biomarkers of Muller Cell Activation in Diabetic Eyes. Invest Ophthalmol Vis Sci (2015) 56(6):3913-8. doi: 10.1167/iovs.15-16554

67. Vujosevic S, Midena E. Retinal layers changes in human preclinical and early clinical diabetic retinopathy support early retinal neuronal and Muller cells alterations. J Diabetes Res (2013) 2013:905058. doi: 10.1155/2013/905058

68. Nork TM, Wallow IH, Sramek SJ, Anderson G. Muller's cell involvement in proliferative diabetic retinopathy. Arch Ophthalmol (1987) 105(10):1424-9. doi: 10.1001/archopht.1987.01060100126042

69. Yanoff M, Sassani JW. 15 - Diabetes Mellitus. In: M Yanoff, JW Sassani, editors. Ocular Pathology, Seventh Edition. London: W.B. Saunders (2015). p. 527-53.e7.

70. De Filippo K, Henderson RB, Laschinger M, Hogg N. Neutrophil chemokines $\mathrm{KC}$ and macrophage-inflammatory protein- 2 are newly synthesized by tissue macrophages using distinct TLR signaling pathways. J Immunol (2008) 180 (6):4308-15. doi: 10.4049/jimmunol.180.6.4308

71. Lange CAK, Stavrakas P, Luhmann UFO, de Silva DJ, Ali RR, Gregor ZJ, et al. Intraocular oxygen distribution in advanced proliferative diabetic retinopathy. Am J Ophthalmol (2011) 152(3):406-12 e3. doi: 10.1016/j.ajo.2011.02.014

72. Tang J, Allen Lee C, Du Y, Sun Y, Pearlman E, Sheibani N, et al. MyD88dependent pathways in leukocytes affect the retina in diabetes. PloS One (2013) 8(7):e68871. doi: 10.1371/journal.pone.0068871

73. Della Sala S, Bertoni G, Somazzi L, Stubbe F, Wilkins AJ. Impaired contrast sensitivity in diabetic patients with and without retinopathy: a new technique for rapid assessment. Br J Ophthalmol (1985) 69(2):136-42. doi: 10.1136/ bjo.69.2.136

74. Sokol S, Moskowitz A, Skarf B, Evans R, Molitch M, Senior B. Contrast sensitivity in diabetics with and without background retinopathy. Arch Ophthalmol (1985) 103(1):51-4. doi: 10.1001/archopht.1985.01050010055018

75. Bloodworth JM Jr. Diabetic retinopathy. Diabetes (1962) 11:1-22.

76. Barber AJ, Lieth E, Khin SA, Antonetti DA, Buchanan AG, Gardner TW. Neural apoptosis in the retina during experimental and human diabetes. Early onset and effect of insulin. J Clin Invest (1998) 102(4):783-91. doi: 10.1172/ JCI2425

77. Liu F, Saul AB, Pichavaram P, Xu Z, Rudraraju M, Somanath PR, et al. Pharmacological Inhibition of Spermine Oxidase Reduces Neurodegeneration and Improves Retinal Function in Diabetic Mice. J Clin Med (2020) 9(2)340. doi: $10.3390 / \mathrm{jcm} 9020340$

78. Sadowitz PD, Hubbard BA, Dabrowiak JC, Goodisman J, Tacka KA, Aktas MK, et al. Kinetics of cisplatin binding to cellular DNA and modulations by thiol-blocking agents and thiol drugs. Drug Metab Dispos (2002) 30(2):18390. doi: $10.1124 / \mathrm{dmd} \cdot 30.2 .183$

79. Narayanan SP, Shosha E C. DP. Spermine oxidase: A promising therapeutic target for neurodegeneration in diabetic retinopathy. Pharmacol Res (2019) 147:104299. doi: 10.1016/j.phrs.2019.104299

Conflict of Interest: The authors declare that the research was conducted in the absence of any commercial or financial relationships that could be construed as a potential conflict of interest.

Copyright (C) 2020 Murata, Noda and Ishida. This is an open-access article distributed under the terms of the Creative Commons Attribution License (CC BY). The use, distribution or reproduction in other forums is permitted, provided the original author(s) and the copyright owner(s) are credited and that the original publication in this journal is cited, in accordance with accepted academic practice. No use, distribution or reproduction is permitted which does not comply with these terms. 